\title{
Mechanical bowel preparation for elective colorectal surgery
}

\author{
J. Lewis ${ }^{1} \cdot$ J. Kinross ${ }^{1}$
}

Received: 4 July 2019 / Accepted: 5 August 2019 / Published online: 30 August 2019

(c) The Author(s) 2019

\section{Abridged abstract [1]}

Background The presence of bowel contents during colorectal surgery has been related to anastomotic leakage, but the belief that mechanical bowel preparation (MBP) is an efficient agent against leakage and infectious complications is based on observational data and expert opinions only.

Objectives To determine the security and effectiveness of MBP on morbidity and mortality in colorectal surgery.

Search methods Publications describing trials of MBP before elective colorectal surgery were sought through searches of MEDLINE, EMBASE, LILACS, IBECS and The Cochrane Library. Searches were performed December $1,2010$.

Selection criteria Randomized controlled trials (RCTs) including participants submitted for elective colorectal surgery.

Main results For the comparison mechanical bowel preparation (Group A) versus no mechanical bowel preparation (Group B) results were: (1) anastomotic leakage for low anterior resection: $8.8 \%$ (38/431) of Group A, compared with $10.3 \%$ (43/415) of Group B; Peto OR 0.88 [0.55, 1.40]. (2) Anastomotic leakage for colonic surgery: $3.0 \%$ (47/1559) of Group A, compared with 3.5\% (56/1588) of Group B; Peto OR $0.85[0.58,1.26]$. (3) Overall anastomotic leakage: $4.4 \%(101 / 2275)$ of Group A, compared with 4.5\% (103/2258) of Group B; Peto OR 0.99 [0.74, 1.31]. (4) Wound infection: $9.6 \%$ (223/2305) of Group A, compared with 8.5\% (196/2290) of Group B; Peto OR 1.16

J. Lewis

james.lewis1@imperial.ac.uk

1 Department of Surgery and Cancer, Imperial College London, London, UK
$[0.95,1.42]$. Sensitivity analyses did not produce any differences in overall results. For the comparison mechanical bowel preparation (A) versus rectal enema (B) results were: (1) anastomotic leakage after rectal surgery: $7.4 \%(8 / 107)$ of Group A, compared with 7.9\% (7/88) of Group B; Peto OR 0.93 [0.34, 2.52]. (2) Anastomotic leakage after colonic surgery: $4.0 \%$ (11/269) of Group A, compared with 2.0\% (6/299) of Group B; Peto OR 2.15 [0.79, 5.84]. (3) Overall anastomotic leakage: $4.4 \%$ (27/601) of Group A, compared with 3.4\% (21/609) of Group B; Peto OR 1.32 [0.74, 2.36]. (4) Wound infection: 9.9\% (60/601) of Group A, compared with 8.0\% (49/609) of Group B; Peto OR 1.26 [0.85, 1.88].

Authors' conclusions There is no statistically significant evidence that patients benefit from mechanical bowel preparation, nor the use of rectal enemas. Further research on patients having elective rectal surgery, below the peritoneal reflection, in whom bowel continuity is restored, and studies with patients having laparoscopic surgery are still warranted.

\section{Commentary}

Recent advances in our understanding of the biological mechanisms underlying anastomotic leak [2] have led to a re-examination of the role of bowel preparation in elective colorectal surgery. MBP is commonly with the aim of reducing peritoneal contamination and to reduce wound infections or the risk of anastomotic dehiscence. Much of the current debate has been fueled by retrospective data from US registries which suggest that oral antibiotics, when used with bowel preparation, reduced surgical morbidity [3]. However, high-quality data on the single use of mechanical bowel preparation are still lacking and as a result, there is significant clinical heterogeneity in its use. The Cochrane review on this topic from 2011 [1] is, therefore, of relevance once more. For this systematic review and meta-analysis, the authors collated both published and unpublished data from 18 heterogenous studies which, at the time of writing, provided the most rigorous data on mechanical bowel 
preparation. In this fourth update of the Cochrane review, including data from 5805 patients, the authors found that mechanical bowel preparation confers no advantage over either no bowel preparation or rectal enema with regard to infectious complications or anastomotic dehiscence. This lack of a statistically significant difference in outcomes holds true for both colonic and rectal resections.

However, the analysis warrants closer inspection. Of the eight studies that calculated sample size, only three were powered to detect differences in anastomotic leak [4-6]. More importantly, there is significant heterogeneity across the clinical studies both in terms of trial design and clinical intervention. For example, a variety of different bowel preparation methods were used including mannitol, bisacodyl, polyethylene glycol, and sodium phosphate. Recent data suggest that the colonic mucosal bacteria vary significantly according to the underlying pathology [7], yet these studies included heterogenous cohorts of patients having surgery for both malignant and benign conditions. Similarly, there was no standardization of surgical approach (three studies report the inclusion of laparoscopic surgery) or post-operative management; in the current era of laparoscopic surgery and enhanced recovery protocols, the results should, therefore, be interpreted with caution.

Perhaps most importantly, a variety of antimicrobial prophylaxis approaches were used which are likely to have confounded many of the central conclusions of the analysis. One study alone includes five intravenous antibiotic combinations [4], some routinely continued antibiotics (gentamicin + metronidazole) post-operatively for 24 [8] or $48 \mathrm{~h}$ [9] (or longer), some used for enteral (sulfamethoxazole/ trimethoprim + metronidazole or doxycycline + metronidazole) antibiotic prophylaxis [5], and one used both enteral (neomycin + metronidazole $24 \mathrm{~h}$ pre-operatively) and intravenous (ceftriaxone + metronidazole at induction) antibiotic prophylaxis [10]. The widely varied use of antibiotics has not been controlled for in this analysis. The authors note that all studies included antibiotic prophylaxis in some form and note the importance of antibiotic prophylaxis in preventing infectious complications, but do not control for the different routes or patterns of administration in analysis.

Although no mention is made in this review of patient acceptability or complications of bowel preparation, some of the individual papers $[4,5,11]$ mention the concerns of patient discomfort, bloating dehydration, nausea, and electrolyte imbalances which detract from the goal of optimal peri-operative physiology. The discomfort of mechanical bowel preparation led to $11 \%$ of patients failing to complete preparation in one study [12], and Moral et al. [10] reported that $35 \%$ of their patients described intense discomfort with mechanical bowel preparation. Platell et al. [6] noted that mechanical bowel preparation with polyethylene glycol resulted in more patient discomfort and nausea than enema preparation and that the mechanical bowel preparation was reported to be significantly worse.

The reviewers state that updates are considered every 2 years, but none has been forthcoming for 8 years. In that time, clinical practice has advanced with many centres in the UK adopting oral antibiotic use. In a more recent Cochrane review by Nelson et al. [13], which explored the use of antimicrobial prophylaxis in colorectal surgery, a significantly reduced risk of surgical site infection was identified when using combined oral and intravenous antibiotic preparation compared to intravenous alone [RR $0.55,95 \%$ CI $0.43-0.71, p=0.0001$, data from 15 randomized controlled trials (RCTs) with 2929 patients] or oral alone (RR $0.52,95 \%$ CI $0.35-0.76, p=0.0003$, data from 9 RCTs with 1990 patients). In fact, Bellows et al. [14] suggest a number needed to treat of 20 when using oral, non-absorbed antibiotics in addition to intravenous prophylaxis to prevent wound infections after colorectal surgery. They do add that they consider mechanical preparation of the bowel an important synergist to antimicrobial preparation to reduce the bacterial burden and improve delivery of antibiotics to the mucosa.

Perhaps the best recent evidence is an analysis of 27,804 subjects in the American College of Surgeons-National Surgical Quality Improvement Program (ACS-NSQIP) database by Klinger et al. [2]. They showed that oral antibiotic bowel preparation is protective against surgical site infection, organ space infection, and anastomotic leak when compared to no preparation. The same was not true for mechanical bowel preparation alone, which showed no significant difference in results to unprepared bowel. The combination of oral antimicrobial and mechanical bowel preparation also reduced the risk of surgical site infection when compared to oral antimicrobial preparation alone. Although this provides some evidence in support of combined mechanical and antimicrobial bowel preparation, we are still lacking a large-scale randomized trial of bowel preparation techniques to answer the question of how to proceed. While there have been several studies into the effect of antimicrobial bowel preparation, none have replicated modern practices with laparoscopic surgery and enhanced recovery after surgery (ERAS) protocols, most are not powered for leak, and they have mostly compared oral with intravenous antimicrobial prophylaxis. Moreover, few have reported mechanisms, side effect data or complications to the patient (e.g., Clostridium difficile infection).

The gut microbiota (the community of prokaryotes and eukaryotes colonising the gastrointestinal tract) varies between individuals, as does the microbiome (the genomic content of the microbiota) [15]. Recent work has implicated the microbiome in the pathogenesis of anastomotic leak and demonstrated that virulent strains of Enterococcus faecalis can take the advantage of a depleted post-operative colonic microbiome and contribute to anastomotic leak via MMP-9 
activation and collagenase expression [1]. Mucosal bacteria therefore represent a legitimate target for peri-operative strategies aiming to reduce risk; however, the best method to target these bacteria is still not established and there remains a lack of precision strategies for targeting nichespecific strains. Moreover, the microbiome has a very broad range of health benefits for the host [16] and a perturbed gut ecology will influence the efficacy and toxicity of the response to adjuvant chemotherapy [17]. The importance of the microbiome is, therefore, nuanced, and it is unclear what harm may result from the attempted eradication of the entire colonic microbiome. It is believed that a diverse flora is an important constituent of health and is therefore counterintuitive to eradicate the entire flora for short-term surgical outcomes when the long-term effects of this is not yet understood.

The 2011 Cochrane review [1] remains the best evidence available on mechanical bowel preparation. However, mechanical bowel preparation is still in widespread clinical practice, despite the discomfort caused, and global targeting of the gut microbiome with antibiotics continues to gain popularity despite the lack of understanding of the role of the microbiome in anastomotic healing. More prospective data are now urgently required on the mechanisms of bowel preparation and the reasons why there are such large inter-individual variations in the response to this therapy. Moreover, we will need properly powered and designed microbiome-based trials to inform clinicians on the optimal way to reduce wound infection and anastomotic leak rates.

Funding This work was co-funded by the NIHR Imperial Biomedical Research Centre (BRC).

\section{Compliance with ethical standards}

Conflict of interest James Kinross is a consultant for Ethicon and Verb Robotics and has received funding from NIHR, H2020 and Intuitive. James Lewis declares that he has no conflict of interest.

Open Access This article is distributed under the terms of the Creative Commons Attribution 4.0 International License (http://creativeco mmons.org/licenses/by/4.0/), which permits unrestricted use, distribution, and reproduction in any medium, provided you give appropriate credit to the original author(s) and the source, provide a link to the Creative Commons license, and indicate if changes were made.

\section{References}

1. Guenaga KF, Matos D, Wille-Jorgensen P (2011) Mechanical bowel preparation for elective colorectal surgery. Cochrane Database Syst Rev 9:CD001544

2. Gaines S, Shao C, Hyman N, Alverdy JC (2018) Gut microbiome influences on anastomotic leak and recurrence rates following colorectal cancer surgery. Br J Surg 105(2):e131-e141
3. Klinger AL, Green H, Monlezun DJ, Beck D, Kann B, Vargas HD et al (2019) The role of bowel preparation in colorectal surgery: results of the 2012-2015 ACS-NSQIP data. Ann Surg 269(4):671-677

4. Contant CM, Hop WC, van't Sant HP, Oostvogel HJ, Smeets HJ, Stassen LP et al (2007) Mechanical bowel preparation for elective colorectal surgery: a multicentre randomised trial. Lancet 370(9605):2112-2117

5. Jung B, Pahlman L, Nystrom PO, Nilsson E, Mechanical Bowel Preparation Study G (2007) Multicentre randomized clinical trial of mechanical bowel preparation in elective colonic resection. $\mathrm{Br}$ J Surg 94(6):689-695

6. Platell C, Barwood N, Makin G (2006) Randomized clinical trial of bowel preparation with a single phosphate enema or polyethylene glycol before elective colorectal surgery. Br J Surg 93(4):427-433

7. Yachida S, Mizutani S, Shiroma H, Shiba S, Nakajima T, Sakamoto $\mathrm{T}$ et al (2019) Metagenomic and metabolomic analyses reveal distinct stage-specific phenotypes of the gut microbiota in colorectal cancer. Nat Med 25(6):968-976

8. Pena-Soria MJ, Mayol JM, Anula-Fernandez R, Arbeo-Escolar A, Fernandez-Represa JA (2007) Mechanical bowel preparation for elective colorectal surgery with primary intraperitoneal anastomosis by a single surgeon: interim analysis of a prospective singleblinded randomized trial. J Gastrointest Surg 11(5):562-567

9. Zmora O, Mahajna A, Bar-Zakai B, Rosin D, Hershko D, Shabtai $M$ et al (2003) Colon and rectal surgery without mechanical bowel preparation: a randomized prospective trial. Ann Surg 237(3):363-367

10. Alcántara Moral M, Serra Aracil X, Bombardó Juncá J, Mora López L, Hernando Tavira R, Ayguavives Garnica I et al (2009) A prospective, randomised, controlled study on the need to mechanically prepare the colon in scheduled colorectal surgery. Cirugía Española (English Edition) 85(1):20-25

11. Scabini S, Rimini E, Romairone E, Scordamaglia R, Damiani G, Pertile D et al (2010) Colon and rectal surgery for cancer without mechanical bowel preparation: one-center randomized prospective trial. World J Surg Oncol 8:35

12. Bretagnol F, Panis Y, Rullier E, Rouanet P, Berdah S, Dousset B et al (2010) Rectal cancer surgery with or without bowel preparation: the French GRECCAR III multicenter single-blinded randomized trial. Ann Surg 252(5):863-868

13. Nelson RL, Gladman E, Barbateskovic M (2014) Antimicrobial prophylaxis for colorectal surgery. Cochrane Database Syst Rev 5:CD001181

14. Bellows CF, Mills KT, Kelly TN, Gagliardi G (2011) Combination of oral non-absorbable and intravenous antibiotics versus intravenous antibiotics alone in the prevention of surgical site infections after colorectal surgery: a meta-analysis of randomized controlled trials. Tech Coloproctol 15(4):385-395

15. Thursby E, Juge $N$ (2017) Introduction to the human gut microbiota. Biochem J 474(11):1823-1836

16. Artis D (2008) Epithelial-cell recognition of commensal bacteria and maintenance of immune homeostasis in the gut. Nat Rev Immunol 8:411

17. Alexander JL, Wilson ID, Teare J, Marchesi JR, Nicholson JK, Kinross JM (2017) Gut microbiota modulation of chemotherapy efficacy and toxicity. Nat Rev Gastroenterol Hepatol 14(6):356-365

Publisher's Note Springer Nature remains neutral with regard to jurisdictional claims in published maps and institutional affiliations. 